\title{
White matter damage in patients with mild cognitive impairment in Parkinson's disease
}

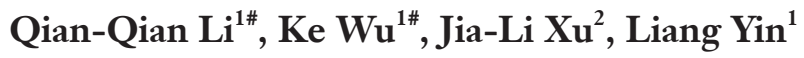 \\ ${ }^{1}$ Department of Neurology, the First Affiliated Hospital of Bengbu Medical College, Bengbu, China; ${ }^{2}$ Department of Imaging, the First Affiliated \\ Hospital of Bengbu Medical College, Bengbu, China
}

Contributions: (I) Conception and design: L Yin, QQ Li; (II) Administrative support: L Yin; (III) Provision of study materials or patients: QQ Li, K $\mathrm{Wu}$; (IV) Collection and assembly of data: JL Xu; (V) Data analysis and interpretation: JL Xu, K Wu; (VI) Manuscript writing: All authors; (VII) Final approval of manuscript: All authors.

\#These authors contributed equally to this work.

Correspondence to: Liang Yin. Department of Neurology, The First Affiliated Hospital of Bengbu Medical College, No. 287 of Changhuai Road, Longzihu District, Bengbu 233004, China. Email: yinliang_sci@163.com.

Background: This study investigates the correlation between white matter structural damage and cognitive impairment in patients with Parkinson's disease (PD) and mild cognitive impairment (MCI).

Methods: A total of 40 patients with $\mathrm{PD}$ were divided into two groups, i.e., a mild cognitive impairment (PD-MCI) and a normal cognitive (PDN) group, and 20 healthy patients were enrolled as the control group. Changes in the white matter structure of patients with PD were evaluated using diffusion tensor imaging (DTI), and cognitive function was evaluated using the Montreal Cognitive Assessment (MoCA) scale and Mini-Mental State Examination (MMSE). Finally, the correlations between the two groups were analyzed.

Results: The Unified Parkinson's Disease Rating Scale score was significantly higher in the PD-MCI than in the PDN group $(\mathrm{P}=0.008)$. The total MoCA and MMSE scores in the PD-MCI group were significantly lower than in the $\mathrm{PDN}$ and control groups $(\mathrm{P}<0.01)$. Patients in PDMCI group were tested by MMSE scale, and the abnormal score rate was $60.0 \%$. Among them, 8 PD patients with normal MMSE total score were found to have mild cognitive impairment by MoCA evaluation. When compared with the PDN and control groups, the MoCA scores for visual space, which is assessed as part of the MoCA scale and generally represents bilateral parietal function; naming; memory; and attention were significantly lower in the PDMCI group $(\mathrm{P}<0.001)$. When compared with the PDN group, the fractional anisotropy $(\mathrm{FA})$ values for the right parietal and left occipital lobes were significantly lower in the PD-MCI group ( $\mathrm{P}=0.005 ; \mathrm{P}=0.018)$. The relationship between MoCA value and right parietal white matter and left occipital white matter was 0.555 , 0.474, respectively. A Pearson's correlation test was conducted to compare the FA values and MoCA scores of the various brain areas in the PD-MCI group and revealed a significant positive correlation between the MoCA score and the white matter of the right parietal and left occipital lobes $(\mathrm{P}<0.01)$.

Conclusions: Patients with PD experience early cognitive impairment, and the MoCA scale can be used for early screening. In addition, the DTI of white matter can highlight early white matter damage. In the current study, the damaged brain areas displayed by DTI were consistent with areas showing decreased MoCA scores and were positively correlated with the severity of PD.

Keywords: Parkinson's disease (PD); early cognitive impairment; white matter damage; diffusion tensor imaging; Montreal Cognitive Assessment scale

Submitted Jun 01, 2021. Accepted for publication Sep 16, 2021.

doi: 10.21037/qims-21-579

View this article at: https://dx.doi.org/10.21037/qims-21-579 


\section{Introduction}

Parkinson's disease (PD) is a chronic degenerative disease of the nervous system, and its pathogenesis is related to dopamine dysfunction in the substantia nigra (1). Its clinical manifestations are static tremor, cogwheel rigidity, bradykinesia, and abnormal posture and gait. To date, studies have found that patients with PD may experience mild cognitive impairment (MCI) and even dementia, as well as other non-motor symptoms $(2,3)$. Once dementia occurs, the quality of life and prognosis of patients with PD are seriously impacted. In the current context, the MCI ranges between what occurs as a result of normal aging and what is caused by dementia. In the future, with earlier and easier diagnosis of PD-MCI, we may be able to effectively slow its progression and prevent dementia (4).

Due to a lack of objective indicators for the diagnosis of MCI, its early detection is difficult. Several studies have revealed a correlation between the onset of cognitive impairment and white matter lesions (WMLs) $(5,6)$. However, the correlation between cognitive function and WMLs is not entirely clear. Diffusion tensor imaging (DTI) can be used to visually and quantitatively evaluate the microstructure of the white matter. Some researchers believe the progression of PD to be closely related to the degree of damage to the white matter fibers (7), and $\mathrm{PD}$ with dementia is a more serious condition than nondementia PD with white matter damage (8). The purpose of this study is to evaluate the changes in the white matter structure of patients with PD using DTI, to evaluate the cognitive function of patients with PD using the Montreal Cognitive Assessment (MoCA) scale and the Mini-Mental State Examination (MMSE), and to further investigate the correlation between white matter structural damage and cognitive impairment in patients with PD-MCI. We present the following article in accordance with the MDAR checklist (available at https://dx.doi.org/10.21037/qims-21-579).

\section{Methods}

\section{Subjects}

A total of 40 patients with PD who were treated in the neurology department of our hospital from January 2019 to March 2021 were enrolled in this study, including 19 males and 21 females with an average age of $66.7 \pm 7.5$ years. The patients were divided into two groups ( $\mathrm{n}=20$ in each) according to their MoCA score, i.e., the normal cognitive function (PDN) and MCI (PD-MCI) groups, and 20 subjects whose gender, age, and cultural backgrounds matched those of the PD groups were enrolled as a control group (MoCA score $\geq 26$ points).

The study was conducted in accordance with the Declaration of Helsinki (as revised in 2013) and was approved by the ethics committee of the First Affiliated Hospital of the Bengbu Medical College (No. 2020-181), and all study subjects signed an informed consent form.

\section{Inclusion criteria}

(I) Diagnostic criteria for PD: The European Federation of Neurological Societies' guidelines (2013) for the diagnosis of PD are as follows: (i) the presence of bradykinesia; (ii) the presence of at least one of the following symptoms: static tremor, myotonia, and postural imbalance; and (iii) the presence of the following three conditions: gradual worsening of the patient's condition, effective treatment with L-dopa, and the course of the disease is $\geq 4 \mathrm{y}$.

(II) Diagnostic criteria for PD with MCI: (i) meeting the diagnostic criteria for PD published by the Movement Disorder Society (9); (ii) slow and progressive cognitive decline confirmed by an informed person or clinician; (iii) decline in cognitive function in accordance with a neuropsychological rating scale or international cognitive rating scale, and a score in at least one cognitive domain of $<1.5 \times$ standard deviation (SD); and (iv) daily life is essentially normal.

(III) Exclusion criteria: (i) Parkinson's syndrome (including vascular, metabolic toxic, infectious, traumatic, and neoplastic syndromes); (ii) patients with PD undergoing surgical treatment; (iii) patients complicated with important organ diseases (such as heart, liver, and kidney disease); (iv) patients with a severe mental disorder who could not cooperate with the treatment; (v) patients unable to undergo magnetic resonance imaging (MRI) scanning due to a metal implant or poor overall body condition; (vi) a standard self-rating depression scale score $\geq 50$; (vii) patients who met the criteria for PD with dementia (PDD; 2013 version); and (viii) patients with other diseases that cause dementia (such as delirium, stroke, depression, metabolic diseases, medication side effects, and trauma).

\section{Research methods}

This study used a combination of a questionnaire survey 
Table 1 Comparison of general data between PD patients with different cognitive function states and control group

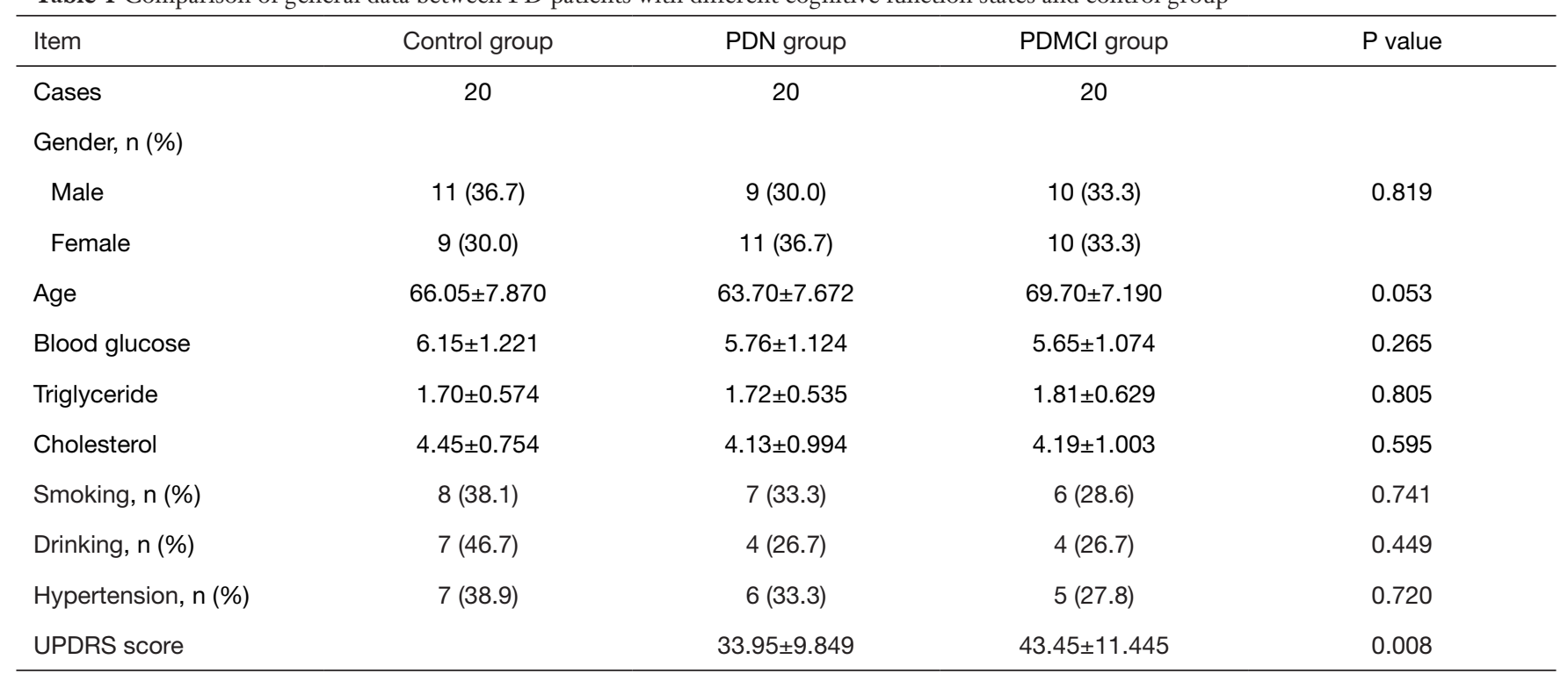

PD, Parkinson's disease; MCl, mild cognitive impairment; UPDRS, Unified Parkinson's Disease Rating Scale.

and clinical examination. All patients and healthy controls were provided with informed consent forms, which they signed as confirmation for inclusion in the study.

General information survey: Information about age, gender, stroke history, blood pressure, blood glucose, triglycerides, cholesterol, smoking, and drinking were recorded.

The Unified Parkinson's Disease Rating Scale (UPDRS) was used to evaluate the condition of all patients with PD.

The Chinese version of the MMSE and MoCA scale were used to evaluate the cognitive function of the subjects. The MMSE scoring criteria were as follows: when uneducated subjects scored $>17$ points, subjects with $<6$ y of education scored $>20$, and subjects with $>6$ y of education scored $>24$ points, the scores were considered normal. A MoCA score of $<26$ indicated cognitive impairment. All the tests were completed by the subjects themselves.

Diffusion tensor imaging: All subjects underwent a head scan with a 3.0T MRI machine (GE Healthcare, $\mathrm{UK}$ ), and the routine sequences included $\mathrm{T} 1$ weighted image (WI), T2WI, fluid-attenuated inversion recovery, diffusion-weighted imaging, and DTI. The DTI images were processed using an Advantage Workstation 4.4 and the Functools image analysis software package. The fractional anisotropy (FA) values of the white matter nerve fibers in different regions of interest (ROIs) were measured, including ROIs selected from the bilateral symmetrical parts of the pons, thalamus, substantia nigra, red nucleus, frontal lobe, parietal lobe, temporal lobe, and occipital lobe.
All data were measured three times by two staff members with the title of deputy chief physician, and the average values were calculated and used as the results.

\section{Statistical analysis}

In this study, the SPSS Statistics version 19.0 software package (IBM, USA) was used for data analysis. Measurement data were expressed as mean \pm SD $(\bar{x} \pm \mathrm{SD})$ and were first subjected to an intergroup comparison using analysis of variance and then subjected to multiple comparisons among the means using the Student-NewmanKeuls q test. The count data were expressed as percentages $(\%)$ and compared using the chi-squared test $\left(\chi^{2}\right)$. Pearson's correlation was used to analyze the associations between the FA values and MoCA scores in each brain area, and $\mathrm{P}<0.05$ was considered statistically significant.

\section{Results}

\section{Comparison of the general data between patients with Parkinson's disease with different cognitive functional states and the control group}

There were no significant differences in age and gender between the PDN, PD-MCI, and control groups $(\mathrm{P}>0.05)$. The UPDRS score was significantly higher in the PD-MCI group than in the $\mathrm{PDN}$ group $(\mathrm{P}<0.05$; see Table 1$)$. 
Table 2 Comparison of total scores of MoCA and MMSE between PD patients with different cognitive function states and control group

\begin{tabular}{lclcc}
\hline Item & Control group & PDN group & PDMCl group & P value \\
\hline MMSE score & $28.35 \pm 1.461$ & $28.80 \pm 1.58$ & $25.35 \pm 3.68^{\#}$ & $<0.01$ \\
MoCA score & $28.40 \pm 1.14$ & $27.50 \pm 1.32$ & $22.75 \pm 1.97^{*}$ & $<0.01$ \\
\hline
\end{tabular}

Compared with the PDN group ${ }^{\#} \mathrm{P}<0.01$, compared with the control group ${ }^{*} \mathrm{P}<0.01$. PD, Parkinson's disease; MoCA, Montreal Cognitive Assessment; MMSE, Mini-Mental State Examination.

Table 3 Comparison of MoCA score of different brain areas between PD patients with different cognitive function states and control group

\begin{tabular}{|c|c|c|c|c|c|}
\hline Characteristic & Control group & PDN group & PDMCI group & $\mathrm{t}$ value & $P$ value \\
\hline Naming & $2.75 \pm 0.444$ & $2.55 \pm 0.510$ & $2.15 \pm 0.366$ & 9.46 & $<0.001$ \\
\hline Memory & $4.70 \pm 0.470$ & $4.60 \pm 0.598$ & $2.80 \pm 1.322$ & 29.49 & $<0.001$ \\
\hline Attention & $5.65 \pm 0.671$ & $5.40 \pm 0.598$ & $4.90 \pm 0.788$ & 6.12 & 0.004 \\
\hline Abstract & $1.90 \pm 0.308$ & $1.85 \pm 0.366$ & $1.85 \pm 0.366$ & 0.14 & 0.872 \\
\hline Directional ability & $5.85 \pm 0.366$ & $5.65 \pm 0.587$ & $5.35 \pm 0.851$ & 3.66 & 0.063 \\
\hline
\end{tabular}

PD, Parkinson's disease; MoCA, Montreal Cognitive Assessment.

Comparison of the total scores on the Montreal Cognitive Assessment scale and Mini-Mental State Examination between patients with Parkinson's disease with different cognitive functional states and the control group

The total scores on the MoCA and MMSE in the PD-MCI group were significantly lower than in the PDN and control groups $(\mathrm{P}<0.01)$, but the difference between the scores in the control and PDN groups was not statistically significant. The patients in the PD-MCI group were tested using the MMSE, and the abnormal score rate was $60.0 \%$, but eight patients with PD with a normal MMSE score were found to have MCI by the MoCA scale. These results suggest that when assessing the cognitive function of patients with $\mathrm{PD}$ patients, the sensitivity of the MoCA scale is better than the MMSE (see Table 2).

\section{Comparison of the Montreal Cognitive Assessment results} for cognitive function in various cognitive domains between the Parkinson's disease groups and the control group

The differences between the PD-MCI, PDN, and control groups in terms of the MoCA scores for visual space, naming, memory, and attention were statistically significant $(\mathrm{P}<0.01$; see Table 3).
Comparison of the fractional anisotropy values between the Parkinson's disease mild cognitive impairment and normal cognitive function groups

When compared with the PDN group, the FA values for the right parietal and left occipital lobes were significantly lower in the PD-MCI group $(\mathrm{P}<0.05)$. However, the differences in the $\mathrm{FA}$ values for the other brain areas were not statistically significant (see Table 4 and Figure 1).

\section{Pearson's correlation analysis between the fractional anisotropy values and the Montreal Cognitive Assessment scores of various brain areas in the PD-MCI group}

An analysis of the FA values and the MoCA scores of various brain areas in the PD-MCI group using Pearson's correlation revealed a significant positive correlation between the MoCA and the right parietal white matter (correlation coefficient: 0.555 ) and between the MoCA and the parietal white matter of the left occipital lobe (correlation coefficient: 0.474 ; $\mathrm{P}<0.01$; see Table 5).

\section{Discussion}

PD can present with motor symptoms and can also be 
Table 4 Comparison among brain regions in PD patients with different cognitive function states

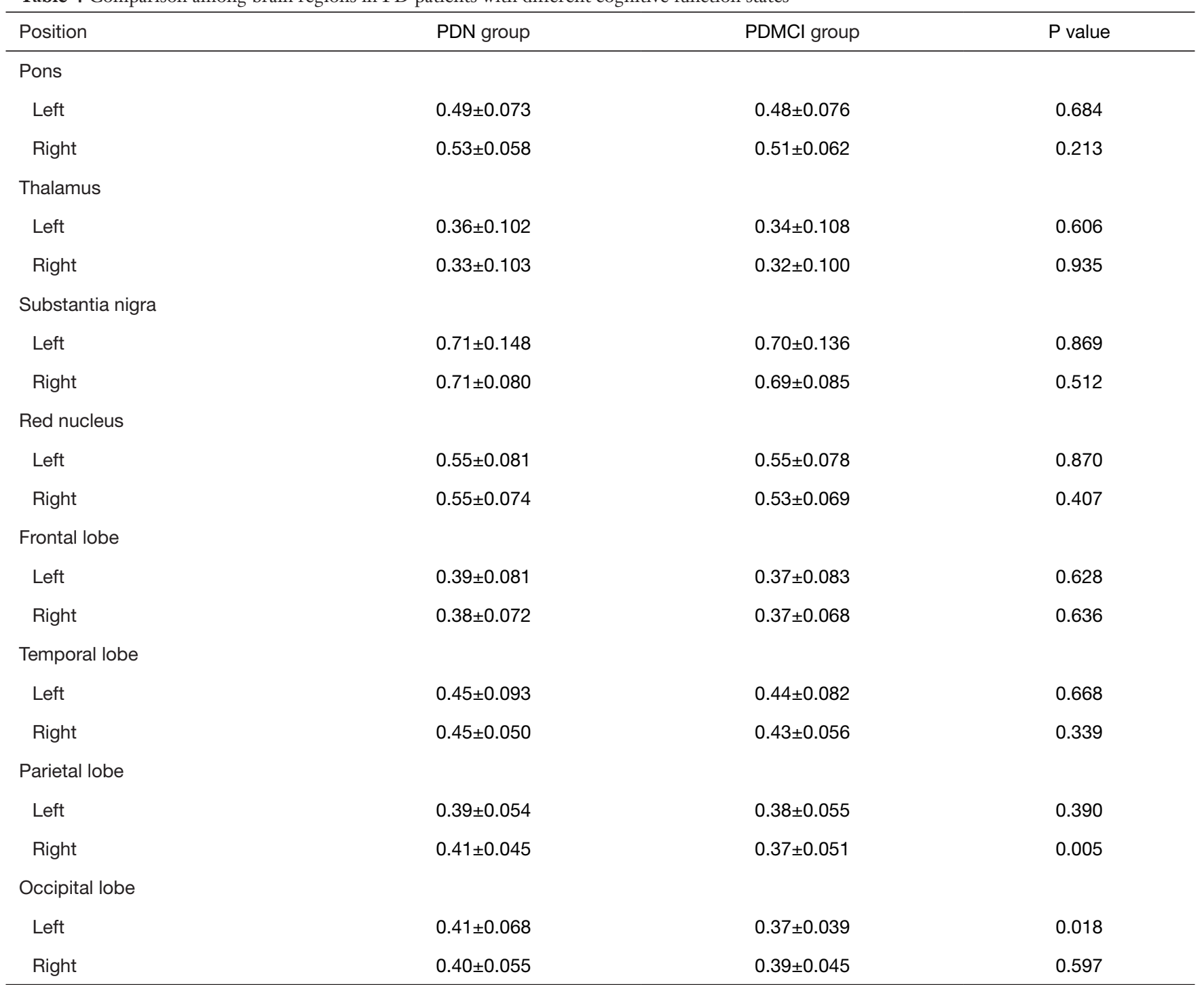

PD, Parkinson's disease.

accompanied by cognitive impairment, sleep disorders, and other non-motor symptoms $(10,11)$. Studies have shown that in people over the age of 65 years, the incidence of PD is $1.7 \%(12,13)$. PD with cognitive impairment has become a popular topic in neuroscience and includes PD-MCI and PDD. The cognitive function of patients with PD-MCI falls between normal cognition and dementia.

By closely focusing on PD-MCI and studying its pathogenesis and risk factors in detail, early detection and intervention should be possible and will assist in controlling or delaying the progression of PD to dementia, thereby improving the quality of life of patients with PDD.
An existing study (14) revealed that PD-MCI can present with impairments in visual space, executive ability, attention, language function, and memory. This study revealed no significant differences in age, gender, blood glucose, and other conventional indicators between the $\mathrm{PD}$ and control groups $(\mathrm{P}>0.05)$, and the UPDRS score of patients with PD gradually increased as cognitive impairment worsened $(\mathrm{P}<0.05)$, suggesting that with the development of Parkinson's motor symptoms, cognitive function will decrease. To some degree, the present study revealed the sensitivity of the MoCA scale and MMSE in evaluating PD-MCI, suggesting the possibility of the early 

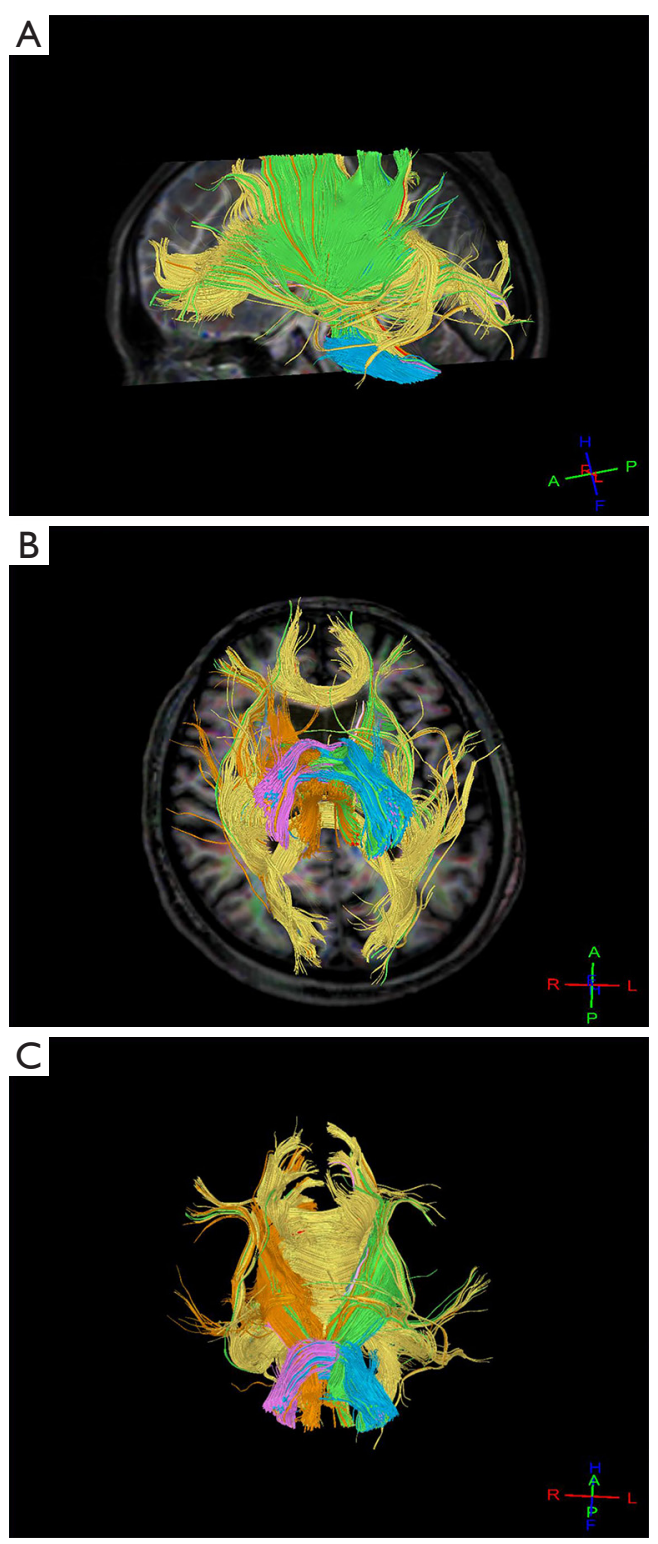

Figure 1 MR DTI shows white matter fiber bundle shape. MR DTI, magnetic resonance diffusion tensor imaging. detection, diagnosis, and determination of the severity of PD-MCI, thereby providing a basis for its early prevention and treatment.

In the current study, patients in the PD-MCI group were tested using the MMSE, with an abnormal score rate of $60.0 \%$. However, eight patients with PD who achieved a normal MMSE score were found to have MCI when using the MoCA scale. This result suggests that when screening the cognitive function of patients with $\mathrm{PD}$, the sensitivity of the MoCA scale is better than the MMSE. To some degree, the MMSE is influenced by educational level, which can cause false positive or false negative results. In addition, memory and attention tests may be too simple, and there are more language items than nonlanguage items. Biundo et al. (15) used the MoCA scale and MMSE to evaluate the cognitive impairment of patients with $\mathrm{PD}$, and the results indicated that both approaches could identify cognitive impairment in patients with PD. However, the MoCA scale is more sensitive in this regard than the MMSE. Some patients with an MMSE score $>26$ had local cognitive impairment affecting memory and visual space. However, a total MoCA score < 26 suggested that patients with a negative MMSE test result may still have MCI, which is supported by the results of the present study. A possible reason for this may be that when compared with the MMSE, the items of abstraction, executive function, reasonably delayed recall, attention, and other cognitive functions are included in the MoCA scale and, accordingly, improve the requirements for testing complex visual spatial, memory, and language abilities.

The mechanism of cognitive impairment caused by WMLs may be as follows: WMLs result in the destruction of the integrity of the white matter fibers and interrupt the connections between the cerebral cortex of the lobes. As a result, the connection between the two cerebral hemispheres becomes abnormal, and the neural circuits

Table 5 Pearson's correlation analysis between FA values and MoCA scores of various brain areas in the PDMCI group

\begin{tabular}{lcc}
\hline Position & Correlation coefficient $(r)$ & $P$ value \\
\hline White matter of right parietal lobe & 0.555 & $<0.01$ \\
White matter of left occipital lobe & 0.474 & $<0.01$ \\
\hline
\end{tabular}

FA, fractional anisotropy; MoCA, Montreal Cognitive Assessment. 
related to emotion and thinking are damaged (16). DTI is a new diffusion imaging technology through which an image is obtained by implementing sensitive gradients in multiple linear directions. The process is a non-invasive testing method that can quantitatively analyze the dispersion of water molecules in cell matter to reveal microscopic changes in white matter fibers in the brain at the cellular and molecular levels (17). In addition, FA is the most sensitive index for reflecting anisotropy between different tissues and can illuminate the state of injury to the nerve fiber tissue. The higher the FA value, the more consistent the fiber shape of the tissue structure. Conversely, when they are closely arranged, structural damage is indicated. The FA value can indirectly show the shape and arrangement of the white matter fiber tracts, which can be used to evaluate the integrity of the white matter microstructure (18). More studies are using DTI to explore changes in the white matter fibers of patients with PD. Braak et al. (19) conducted a DTI analysis of the striatum in patients with $\mathrm{PD}$ and healthy volunteers, and the results revealed a significantly lower FA value for the striatum in patients with PD. Li et al. (20) studied the relationship between PD with depression and the thalamus using whole-brain analysis, and the results revealed that the FA values for the bilateral medial and dorsal thalamus were lower in patients with PD and depression than in healthy patients and that the decrease in FA in the thalamus may reflect a decrease in white matter tracts.

In the current study, when compared with the control and PDN groups, the MoCA scores revealed that patients with PD-MCI exhibited a functional decline in visual space, naming, memory, and attention, and DTI detection showed significantly decreased FA values for the right parietal and left occipital lobes, and the differences were statistically significant. The Pearson's correlation analysis between the FA values and the MoCA scores for various brain areas in the PD-MCI group revealed a significant positive correlation between the MoCA score and the FA values of the white matter of the right parietal and left occipital lobes $(\mathrm{P}<0.01)$. Another study (21) adopted arterial spin labeling technology to detect the cerebral blood flow in patients with PD-MCI, and the results showed that when compared with healthy subjects, the blood flow was lower in the posterior parietal lobe, the parieto-occipital junction area, and the precuneus lobe, suggesting that the decline in cognitive function was related to abnormal brain function in these areas. Another study (22) indicated that when compared with patients with normal cognitive function, patients with cognitive impairment exhibited decreased metabolism in the parieto-occipital lobe and bilateral frontal and middle lobes. These results comprehensively suggest that the posterior parietal lobe and parieto-occipital junction may be linked to episodic memory and visual space. These findings are supported by the current study.

The present study had some limitations. (I) The sample size was small, and no dynamic change study was conducted. It is expected that the correlation between the deterioration in cognitive function and the aggravation of white matter damage in the later stage will be further investigated in future studies. (II) This study only focused on the relationship between white matter damage and cognitive impairment. Additional research should include the relationship between changes in gray matter volume and cognitive impairment in patients with PD-MCI. (III) For ethical reasons, DTI of the white matter was not conducted in the control group.

In summary, many patients with PD experience early cognitive impairment. The MoCA scale can be used for early screening, and the DTI of white matter can show early white matter damage. The damaged brain areas displayed by DTI are consistent with the areas with decreased MoCA scores and are positively correlated with the severity of PD. Therefore, the MoCA scale combined with the DTI of the white matter can provide an important clinical basis for the early diagnosis of PD-MCI.

\section{Acknowledgments}

We would like to acknowledge the hard and dedicated work of all the staff that implemented the intervention, evaluation components and assistance with English language editing of the study.

Funding: This work was supported by the Key Natural Science Project of Bengbu Medical College (BYKY2019041ZD); Bengbu Social Science Planning General Project (BB19C055) and Key Special Project of Translational Medicine in Bengbu Medical College (BYTM2019030).

\section{Footnote}

Reporting Checklist: The authors have completed the MDAR checklist. Available at https://dx.doi.org/10.21037/ qims-21-579

Conflicts of Interest: All authors have completed the ICMJE uniform disclosure form (available at https://dx.doi. 
org/10.21037/qims-21-579). The authors have no conflicts of interest to declare.

Ethical Statement: The authors are accountable for all aspects of the work in ensuring that questions related to the accuracy or integrity of any part of the work are appropriately investigated and resolved. The study was conducted in accordance with the Declaration of Helsinki (as was revised in 2013). The study was approved by Ethics Committee of the First Affiliated Hospital of Bengbu Medical College (No.2020-181) and all subjects provided a signed informed consent form for inclusion in the study.

Open Access Statement: This is an Open Access article distributed in accordance with the Creative Commons Attribution-NonCommercial-NoDerivs 4.0 International License (CC BY-NC-ND 4.0), which permits the noncommercial replication and distribution of the article with the strict proviso that no changes or edits are made and the original work is properly cited (including links to both the formal publication through the relevant DOI and the license). See: https://creativecommons.org/licenses/by-nc-nd/4.0/.

\section{References}

1. Cuenca L, Gil-Martinez AL, Cano-Fernandez L, SanchezRodrigo C, Estrada C, Fernandez-Villalba E, Herrero MT. Parkinson's disease: a short story of 200 years. Histol Histopathol 2019;34:573-91.

2. Litvan I, Aarsland D, Adler CH, Goldman JG, Kulisevsky J, Mollenhauer B, Rodriguez-Oroz MC, Tröster AI, Weintraub D. MDS Task Force on mild cognitive impairment in Parkinson's disease: critical review of PDMCI. Mov Disord 2011;26:1814-24.

3. Sollinger AB, Goldstein FC, Lah JJ, Levey AI, Factor SA. Mild cognitive impairment in Parkinson's disease: subtypes and motor characteristics. Parkinsonism Relat Disord 2010;16:177-80.

4. Emre M, Aarsland D, Brown R, Burn DJ, Duyckaerts C, Mizuno Y, et al. Clinical diagnostic criteria for dementia associated with Parkinson's disease. Mov Disord 2007;22:1689-707; quiz 1837.

5. Kuhn T, Becerra S, Duncan J, Spivak N, Dang BH, Habelhah B, Mahdavi KD, Mamoun M, Whitney M, Pereles FS, Bystritsky A, Jordan SE. Translating stateof-the-art brain magnetic resonance imaging (MRI) techniques into clinical practice: multimodal MRI differentiates dementia subtypes in a traditional clinical setting. Quant Imaging Med Surg 2021;11:4056-73.

6. Louis ED, Brickman AM, DeCarli C, Small SA, Marder K, Schupf N, Brown TR. Quantitative brain measurements in community-dwelling elderly persons with mild parkinsonian signs. Arch Neurol 2008;65:1649-54.

7. Guimarães RP, Campos BM, de Rezende TJ, Piovesana L, Azevedo PC, Amato-Filho AC, Cendes F, D'Abreu A. Is Diffusion Tensor Imaging a Good Biomarker for Early Parkinson's Disease? Front Neurol 2018;9:626.

8. Bledsoe IO, Stebbins GT, Merkitch D, Goldman JG. White matter abnormalities in the corpus callosum with cognitive impairment in Parkinson disease. Neurology 2018;91:e2244-55.

9. Litvan I, Goldman JG, Tröster AI, Schmand BA, Weintraub D, Petersen RC, Mollenhauer B, Adler CH, Marder K, Williams-Gray CH, Aarsland D, Kulisevsky J, Rodriguez-Oroz MC, Burn DJ, Barker RA, Emre M. Diagnostic criteria for mild cognitive impairment in Parkinson's disease: Movement Disorder Society Task Force guidelines. Mov Disord 2012;27:349-56.

10. Hoogland J, Boel JA, de Bie RMA, Schmand BA, Geskus RB, Dalrymple-Alford JC, Marras C, Adler CH, Weintraub D, Junque C, Pedersen KF, Mollenhauer B, Goldman JG, Tröster AI, Burn DJ, Litvan I, Geurtsen GJ; MDS Study Group "Validation of Mild Cognitive Impairment in Parkinson Disease". Risk of Parkinson's disease dementia related to level I MDS PD-MCI. Mov Disord 2019;34:430-5.

11. Seppi K, Ray Chaudhuri K, Coelho M, Fox SH, Katzenschlager R, Perez Lloret S, Weintraub D, Sampaio C; the collaborators of the Parkinson's Disease Update on Non-Motor Symptoms Study Group on behalf of the Movement Disorders Society Evidence-Based Medicine Committee. Update on treatments for nonmotor symptoms of Parkinson's disease-an evidence-based medicine review. Mov Disord 2019;34:180-98.

12. Zhang ZX, Roman GC, Hong Z, Wu CB, Qu QM, Huang JB, Zhou B, Geng ZP, Wu JX, Wen HB, Zhao H, Zahner GE. Parkinson's disease in China: prevalence in Beijing, Xian, and Shanghai. Lancet 2005;365:595-7.

13. Hugo J, Ganguli M. Dementia and cognitive impairment: epidemiology, diagnosis, and treatment. Clin Geriatr Med 2014;30:421-42.

14. Koshimori Y, Segura B, Christopher L, Lobaugh N, DuffCanning S, Mizrahi R, Hamani C, Lang AE, Aminian K, Houle S, Strafella AP. Imaging changes associated with cognitive abnormalities in Parkinson's disease. Brain Struct Funct 2015;220:2249-61. 
15. Biundo R, Weis L, Facchini S, Formento-Dojot $\mathrm{P}$, Vallelunga A, Pilleri M, Antonini A. Cognitive profiling of Parkinson disease patients with mild cognitive impairment and dementia. Parkinsonism Relat Disord 2014;20:394-9.

16. Filley CM, Fields RD. White matter and cognition: making the connection. J Neurophysiol 2016;116:2093-104.

17. Basser PJ, Mattiello J, LeBihan D. MR diffusion tensor spectroscopy and imaging. Biophys J 1994;66:259-67.

18. Arfanakis K, Haughton VM, Carew JD, Rogers BP, Dempsey RJ, Meyerand ME. Diffusion tensor MR imaging in diffuse axonal injury. AJNR Am J Neuroradiol 2002;23:794-802.

19. Braak H, Del Tredici K, Rüb U, de Vos RA, Jansen Steur EN, Braak E. Staging of brain pathology related to sporadic Parkinson's disease. Neurobiol Aging

Cite this article as: $\mathrm{Li} \mathrm{QQ}, \mathrm{Wu} \mathrm{K}, \mathrm{Xu} \mathrm{JL}$, Yin L. White matter damage in patients with mild cognitive impairment in Parkinson's disease. Quant Imaging Med Surg 2022;12(2):12901298. doi: 10.21037/qims-21-579
2003;24:197-211.

20. Li W, Liu J, Skidmore F, Liu Y, Tian J, Li K. White matter microstructure changes in the thalamus in Parkinson disease with depression: A diffusion tensor MR imaging study. AJNR Am J Neuroradiol 2010;31:1861-6.

21. Shang S, Wu J, Chen YC, Chen H, Zhang H, Dou W, Wang P, Cao X, Yin X. Aberrant cerebral perfusion pattern in amnestic mild cognitive impairment and Parkinson's disease with mild cognitive impairment: a comparative arterial spin labeling study. Quant Imaging Med Surg 2021;11:3082-97.

22. Matsui H, Udaka F, Miyoshi T, Hara N, Tamura A, Oda M, Kubori T, Nishinaka K, Kameyama M. Frontal assessment battery and brain perfusion image in Parkinson's disease. J Geriatr Psychiatry Neurol 2006;19:41-5. 